Revue d'histoire de l'Amérique française

DE REVUE D.HISTOIRE DE L'AMÉRIQUE FRANÇAISE

\title{
Représentations et stratégies sociales d'un marchand étranger à Québec : le journal de Johann Heinrich Juncken (septembre 1788 - mai 1789)
}

\section{Marcelle Cinq-Mars}

Volume 44, numéro 4, printemps 1991

URI : https://id.erudit.org/iderudit/304924ar

DOI : https://doi.org/10.7202/304924ar

Aller au sommaire du numéro

Éditeur(s)

Institut d'histoire de l'Amérique française

ISSN

0035-2357 (imprimé)

1492-1383 (numérique)

Découvrir la revue

Citer cet article

Cinq-Mars, M. (1991). Représentations et stratégies sociales d'un marchand étranger à Québec : le journal de Johann Heinrich Juncken (septembre 1788 mai 1789). Revue d'histoire de l'Amérique française, 44(4), 549-566.

https://doi.org/10.7202/304924ar
Résumé de l'article

Récemment arrivé à Québec, le marchand loyaliste d'origine allemande Johann Heinrich Juncken (c. 1735-1802) doit repartir à zéro. L’analyse de son journal personnel révèle sa mentalité et sa stratégie d'intégration. Les notes météorologiques quotidiennes l'aident à s'acclimater. Ses vêtements et sa culture générale lui permettent de s'identifier au groupe des marchands britanniques. Il utilise les actions charitables pour consolider sa position sociale. Ses préjugés contre les Canadiens français relèvent également de sa stratégie d'insertion. 


\title{
REPRÉSENTATIONS ET STRATÉGIES SOCIALES D'UN MARCHAND ÉTRANGER À QUÉBEC: LE JOURNAL DE JOHANN HEINRICH JUNCKEN (SEPTEMBRE 1788 - MAI 1789) $^{1}$
}

\author{
MARCELLE CINQ-MARS \\ Département d' histoire \\ Université Laval
}

\section{RÉSUMÉ}

Récemment arrivé à Québec, le marchand loyaliste d'origine allemande Johann Heinrich Juncken (c. 1735-1802) doit repartir à zéro. L'analyse de son journal personnel révèle sa mentalité et sa stratégie d'intégration. Les notes météorologiques quotidiennes l'aident à s'acclimater. Ses vêtements et sa culture générale lui permettent de s'identifier au groupe des marchands britanniques. Il utilise les actions charitables pour consolider sa position sociale. Ses préjugés contre les Canadiens français relèvent également de sa stratégie d'insertion.

\begin{abstract}
Having emigrated to Québec city in 1787, Johann Heinrich Juncken (c. 1735-1802) a Loyalist merchant of German Origin, must begin life anew. The analysis of his diary reveals his mentality and his strategy for social integration. The daily notes about the weather are a way to familiarize himself with the country. His clothing and his culture help him to identify with the group of British merchants ans his charitable actions also serve to consolidate his social position. His prejudices against French Canadians are also part of the same strategy.
\end{abstract}

\section{DE LA BIOGRAPHIE À L'HISTOIRE SOCIALE DE L'INDIVIDU}

Depuis déjà plusieurs années, les historiens ont redécouvert la richesse des sources personnelles et des individus qui les produisent. Le renouveau d'intérêt pour ces sources s'inscrit dans une remise en question de l'histoire quantitative ou sérielle qui fait généralement

1 Cet article est tiré d'un mémoire de maîtrise présenté à l'Université Laval au printemps de 1990. Je tiens à remercier tout spécialement monsieur Laurier Turgeon qui, en plus de diriger mon mémoire, a bien voulu me conseiller pour cet article. Je remercie également Rénald Lessard des Archives nationales du Québec qui m'a signalé l'existence de ce journal. La préparation d'une édition critique du manuscrit est en cours.

RHAF, vol. 44, n 4, printemps 1991 
abstraction de l'individu. Comme le dit l'historien Lawrence Stone: «Bien des historiens pensent désormais que la culture du groupe et même le vouloir de l'individu sont en puissance des causes de changements au moins aussi importantes que les forces impersonnelles de la production matérielle et de la croissance démographique.» ${ }^{2} \mathrm{C}^{\prime} e s t$ pourquoi les chercheurs prennent leurs distances par rapport à l'histoire économique et sociale qui prétend reconstituer le passé de façon globale et se tournent davantage vers l'étude de l'individu et des relations qu'il entretient avec son ou ses groupes d'appartenance, vers une histoire à petite échelle qu'on appelle parfois la micro-histoire.

Au Canada et au Québec, il y a une longue et forte tradition biographique. Le Dictionnaire biographique du Canada, projet d'envergure amorcé au cours des années 1960 , vise à faire connaître les figures marquantes de l'histoire canadienne. Ses articles offrent une synthèse de la vie des personnages, de la naissance à la mort, et mettent l'accent sur leurs pratiques professionnelles et publiques. Bon nombre de thèses et d'ouvrages se présentent également comme des biographies, d'hommes politiques surtout, mais aussi de marchands, ce qui nous intéresse plus particulièrement ${ }^{3}$. Ces «biographiessynthèses» qui privilégient les espaces du dehors ne permettent pas d'accéder à l'intimité du quotidien qui, pourtant, modèle la vie à la fois professionnelle et personnelle des individus.

Les historiens européens ont privilégié davantage cette approche et ils ont révélé l'immense potentiel des journaux personnels. Madeleine Foisil, par exemple, a analysé la vie quotidienne de Pierre de Gouberville ${ }^{4}$, gentilhomme français du XVI ${ }^{\mathrm{e}}$ siècle, en reconstituant minutieusement la trame de sa vie de tous les jours à l'aide de son journal. Son étude récente du journal de Jean Héroard, médecin de Louis XIII, s'inspire de la même méthodes. De même, Elizabeth

2 Lawrence Stone, «Retour au récit, ou réflexions sur une nouvelle vieille histoire», Le Débat, 2,4 (sept. 1980): 124.

3 C'est le cas des études de David Roberts, George Allsopp: Quebec Merchant 1733 1805, thèse de M.A., Queen's University, 1974, et de Hilda Neatby, «Pierre Guy: a Montreal Merchant of the Eighteenth Century", Eighteenth-Century Studies, 5,2 (hiver 1971): 224-242. Même les travaux plus récents ne se préoccupent que du commerce et des aspects professionnels de la vie des marchands. C'est le cas de ceux de Louis Michel, «Un marchand rural en NouvelleFrance. François-Augustin Bailly de Messein 1709-1771", Revue d'histoire de l'Amérique française (ci-après RHAF), 33,2 (sept. 1979): 215-262; de Claude Desrosiers, «Un aperçu des habitudes de consommation de la clientèle de Joseph Cartier, marchand général à SaintHyacinthe à la fin du XVIII" siècle», Communications historiques (1984): 91-110; et de Lise StGeorges, «Commerce, crédit et transactions foncières: pratique de la communauté marchande du bourg de l'Assomption, 1748-1791", RIIAF, 39,3 (hiver 1986): 323-343.

$4 \quad$ Madeleine Foisil, Le Sire de Gouberville (Paris, Éd. Aubier Montaigne, 1980), 287 p.

5 Madeleine Foisil, dir., Journal de Jean Héroard, médecin de Louis XIII (Paris, Fayard, 1989), $3118 \mathrm{p}$. 
Bourcier a étudié plusieurs journaux privés anglais du $\mathrm{XVII}^{e}$ siècle $^{6}$. Ces historiennes ont su exploiter les sources intimes pour reconstituer la vie d'un individu, pour «voir se dérouler le fil du temps, le rythme d'une vie: vivre la platitude de la vie quotidienne» ${ }^{7}$. Mais ces travaux s'inscrivent encore nettement dans la tradition biographique: ils tentent de reconstituer la vie entière de l'individu et de la présenter comme un modèle à suivre ou à ne pas suivre. De plus, ils sombrent souvent dans la description des détails insignifiants, dans la simple narration de récits de vie, bref, dans la «platitude» tout court.

Dans le courant plus largement inspiré de l'histoire sociale, des chercheurs tels que Carlo Ginzburg ${ }^{8}$, Joël Cornette ${ }^{9}$ et Franco Ferrarotti ${ }^{10}$, soutiennent que l'étude d'un cas particulier permet de mieux connaître le fonctionnement social. Il n'est pas question de prétendre que tel ou tel individu est représentatif de tel ou tel groupe, ni de soutenir qu'il est le reflet de sa société. Ces auteurs étudient les individus plutôt pour appréhender la réalité sociale dans laquelle ceuxci évoluent et pour éclairer leurs rapports avec la société. Dans cette perspective, il semble plus juste et plus pertinent de considérer ces écrits personnels comme autant de représentations individuelles et non pas comme des témoignages historiques sur une société donnée car, comme le rappelle Ferrarotti, chaque individu «lit le groupe à partir de sa perspective individuelle ${ }^{11}$. Pour ce faire, l'historien doit se ranger du côté de son objet d'étude, voire aux côtés de l'écrivant. Il peut ainsi apprécier à sa juste valeur, d'une part, la spécificité et la richesse du journal personnel comme source historique et, d'autre part, l'écrivant comme acteur social faisant représentation de lui-même et de son milieu, faisant donc une mise en scène de son propre «jeu» social.

Dans cet article, nous voulons expliquer la fonction des représentations et des mises en scène que fait l'individu à l'aide de l'écriture du journal personnel. Notre principale préoccupation est de comprendre le journal pour ce qu'il est et pour ce qu'il fait. Pour atteindre cet objectif, nous nous sommes contentée d'étudier un seul journal, celui d'Henry Juncken, marchand loyaliste d'origine allemande ins-

\footnotetext{
1976), $496 \mathrm{p}$

7 Madeleine Foisil, op. cit., 30.

8 Carlo Ginzburg, Le fromage et les vers (Paris, Flammarion, 1980).

9 Joël Cornette, "La personne, l'histoire et le récit: le destin de Benoît Lacombe propriétaire, négociant et révolutionnaire (1783-1819)", Revue d' histoire moderne et contemporaine (ci-après RHMC), 30,2 (avril-juin 1985): 561-590.

10 Franco Ferrarotti, Histoire et histoires de vie: la méthode biographique dans les sciences sociales (Paris, Librairie des Méridiens, 1983), 195 p.

11 Ibid., 62.
} 
tallé à Québec à la fin du XVIII ${ }^{\mathrm{e}}$ siècle. L'étude d'un corpus de journaux aurait permis de faire ressortir des similarités et des constantes, mais elle risquait justement de nous faire négliger les particularités de chacun d'entre eux. Or, ce sont précisément ces particularités qui nous intéressent. Chaque journal n'a-t-il pas d'ailleurs une fonction spécifique qui répond aux besoins d'un individu à un moment donné? Dans le cas d'Henry Juncken, nous pensons que son journal joue le rôle d'un miroir qui lui permet de s'observer, de se mettre en scène et d'étudier son comportement pour ainsi faciliter son intégration dans sa société adoptive. Nous avons privilégié la vie sociale plutôt que l'activité économique du marchand parce que c'est essentiellement de cela dont il est question dans le journal. Sans doute était-il conscient que sa réussite matérielle dépendait de son intégration sociale.

\section{LE JOURNAL PERSONNEL D'HENRY JUNCKEN: CONTENU ET SENS}

Henry Juncken (1735-1802) est né à Durlach, duché de BadeWurtemberg, situé dans le sud-ouest de l'Allemagne. Il émigre en Amérique à l'âge de 18 ans en compagnie de 107 compatriotes et débarque à Philadelphie en 1753 où il épouse une veuve, Anna Barbara $^{12}$. Sa vie s'écoule paisiblement jusqu'à l'époque de la guerre d'Indépendance américaine. Il se range du côté des Loyalistes et, reconnu coupable de haute trahison, il est emprisonné à Reading. Le premier avril 1779, les autorités américaines saisissent sa maison et sa terre de 115 acres situées dans le township de Springfields, sur la route reliant Philadelphie à Germantown. Ses biens, évalués à 1710 livres, sont vendus à l'encan ${ }^{13}$. Dépouillé, il se réfugie à Londres ${ }^{14}$ et, par la suite, voulant profiter des possibilités offertes par le gouvernement britannique en Canada, il décide de s'installer à Québec, en septembre 1787. Il recommence alors sa vie à l'âge de 52 ans. Tout est à refaire: fonder un commerce, s'adapter au pays et s'insérer dans une nouvelle société. Son âge avancé et sa relative insécurité matérielle expliquent en partie l'empressement et le zèle dont il fait preuve dans sa sociabilité. En 1793, Henry Juncken est frappé de paralysie et ne peut plus

12 Ralph Beaver Strassburger, Pennsylvania German Pionners: a Publication of the Original Lists of Arrivals in the Port of Philadelphia from 1727 to 1808. Vol. 1: 1727-1775 (Pennsylvania, German Society, 1934): 531-536.

13 Dossier Henry Juncken, Pennsylvania Historical and Museum Commission, Records of Pennsylvania's Revolutionary Governments - Forfeited Estates files, 1777-1790, RG 27.

14 Les renseignements sur ses activités et ses déboires aux États-Unis se trouvent ici et là dans les cinq réclamations qu'il rédige et envoie au gouvernement britannique pour obtenir un dédommagement. Archives nationales du Canada (ci-après ANC), Audit Papers, bob. 2, 131. 
écrire $^{15}$. Il est inhumé à Québec le 20 octobre $1802^{16}$. Sa femme et ses neveux retournent alors vivre dans la région de Philadelphie.

Henry Juncken nous serait sans doute totalement inconnu s'il n'avait pas écrit un journal personnel qui a eu, en plus, la chance d'être considéré comme un document digne de conservation. Le manuscrit fut cédé aux Archives nationales du Québec par la Literary and Historical Society of Quebec qui l'avait acquis lors d'une vente aux enchères à la fin du XIX ${ }^{e}$ siècle $^{17}$. Ce journal, rédigé en anglais, n'est pas un simple livre de raison: il fournit maints détails sur les gestes et les pensées de son auteur. On y découvre les déplacements, les transactions commerciales et financières, les événements et les rencontres qui rythment la vie quotidienne du marchand. Au fil des jours, Henry Juncken a minutieusement noté à peu près toutes les activités, banales et moins banales, qui balisent sa vie.

Il existe plusieurs catégories de journaux. Le journal émerge au $\mathrm{XVI}^{\mathrm{e}}$ siècle et il est alors personnel et externe: bien que relié à l'idée du «moi», il s'attarde plutôt à la relation de cette entité avec l'Autre et avec le monde extérieur. Ce n'est qu'au XIX ${ }^{\mathrm{e}}$ siècle qu'apparaissent les premiers véritables journaux intimes, de plus en plus centrés sur le «moi», c'est-à-dire sur l'écrivant et sa relation avec lui-même ${ }^{18}$. Le journal de Juncken, écrit dans une période de transition, semble se situer entre ces deux catégories: s'il ne laisse place qu'à de très rares commentaires sur ses propres réflexions et comportements, l'importance qu'il accorde aux éléments extérieurs trouve son origine au plus profond de lui-même. Bien qu'il soit relativement aisé de classer les journaux selon leur nature, il n'en va pas de même de leurs fonctions, tellement celles-ci sont spécifiques. En effet, chaque journal répond aux besoins et aux espérances de son rédacteur. Il faut chercher les fonctions que l'écrivant lui assigne pour retrouver les motivations profondes de celui-ci. Souvent, c'est un élément déclencheur, un traumatisme, qui pousse l'individu à entreprendre une telle rédaction.

Dans le cas de Juncken, le journal personnel joue un rôle thérapeutique, c'est-à-dire qu'il vise à guérir des blessures profondes et

15 ANQ-Q, Greffe de Descheneaux, 8 novembre 1793, Testament d'Henry Juncken.

16 ANQ-Q, Fonds Congregation of the English Church.

17 C'est en cherchant dans les archives de la Literary and Historical Society of Quebec, que j'ai obtenu ces renseignements, grâce à l'aimable collaboration de l'archiviste.

18 J. Bensman et R. Lilienfeld, Between Public and Private: the Lost Boundaries of the Self (New York, The Free Press, 1979). Voir aussi Pierre Hébert, Le journal intime au Québec: structure, évolution, réception (Montréal, Fides, 1988), 17-18. Les journaux d'officiers, très nombreux entre 1759 et 1820 , sont surtout centrés sur les événements militaires. En revanche, les journaux rédigés vers la fin du $\mathrm{XIX}^{\mathrm{c}}$ siècle portent presqu'exclusivement sur la vie intime de leurs auteurs. 
encore fraîches. Ses problèmes au moment de la Révolution américaine découlaient de ses prises de positions politiques. Accusé de trahison, il voit sa vie publique remise en cause, car c'est bien celleci qu'on détruit en le dépossédant de ses biens et de sa personne sociale. Son journal lui sert donc vraisemblablement de mémoire, de carnet de bord et de confident l'aidant à consigner son destin. Comme ses problèmes sont nés de sa vie publique, il doit sentir la nécessité de surveiller continuellement ses moindres faits et gestes pour éviter de nouvelles mésaventures. Voilà pourquoi son journal personnel porte essentiellement sur sa vie publique, celle qui est à l'origine de ses malheurs et qu'il lui faut refaire, plutôt que sur sa vie privée qui est demeurée relativement intacte. C'est également parce qu'il a été profondément meurtri par l'Autre, touché au plus profond de lui-même, qu'il porte une telle attention à ses relations. L'artefact de vie que Juncken nous a laissé est celui d'un réfugié politique et aussi celui d'un étranger qui s'approprie, au fil des jours, sa ville adoptive.

Le «Day Book $F{ }^{19}$ est le seul volume qui nous soit parvenu d'une série qui devait en compter au moins six autres, cinq le précédant et un le suivant. Il s'agit d'un manuscrit opistographe qui couvre neuf mois, de septembre 1788 à mai 1789, et comprend 367 pages de texte extrêmement serré. Certes, il aurait été préférable d'avoir un plus large extrait du journal. Mais, au cours de ces neuf mois d'écriture assidue, Juncken n'a pas omis une seule journée. Le texte ne compte pas moins de 8200 lignes manuscrites pour une moyenne quotidienne de 30 lignes. Cette source inédite offre la possibilité d'effectuer une très bonne micro-étude. Loin d'être un obstacle, la chronologie restreinte s'accorde bien avec les objectifs de cette étude qui doit nécessairement réduire le champ temporel pour approfondir l'analyse. Cet extrait est d'autant plus intéressant qu'il date d'une période importante de la vie de son auteur. En effet, au moment de la rédaction du cahier en question, celui-ci vient à peine de s'installer à Québec. Le regard est donc encore curieux; c'est celui d'un observateur qui, découvrant une réalité nouvelle, note encore abondamment ses expériences, ses impressions et ses réflexions sur la société québécoise.

L'étude du manuscrit fut un véritable travail de moine compte tenu des nombreuses fautes de syntaxe et d'orthographe. Bien qu'écrit avec des mots anglais, le texte est parsemé de constructions calquées sur l'allemand. Pour faciliter la lecture des citations, nous les avons traduites en corrigeant et en actualisant le vocabulaire, tout en respectant la pensée et le sens du texte original. Le même souci a guidé

19 C'est le titre que Juncken a lui-même donné à son journal. 
notre analyse des comportements de Juncken. Le fait qu'il n'a pas jugé bon de commenter tous ses gestes, de livrer des réflexions sur sa démarche d'insertion sociale, un trait typique des journaux de cette époque, ne doit pas empêcher l'historien d'interpréter les conduites, de s'efforcer de comprendre le sens implicite et profond des comportements.

\section{LES ÉLÉMENTS PHYSIQUES:}

\section{LE TEMPS, LA VILLE ET LA MAISON}

Le marchand Juncken multiplie les notations sur le monde matériel qui l'entoure. Il est très attentif aux conditions météorologiques, au milieu géographique et à l'aménagement urbain qu'il ponctue de repères. Pour lui, la connaissance et la maîtrise de ce nouvel environnement représente la première condition de son intégration professionnelle et sociale.

Chacune des entrées de son journal personnel renferme au moins une référence au temps qu'il fait. Ces notations servent d'aidemémoire au diariste. Arrivé à Québec depuis seulement onze mois, il a encore tout à apprendre sur les caprices du cycle saisonnier. Les données qu'il enregistre lui permettent de comparer ses observations d'une année à l'autre et de se familiariser rapidement avec les particularités du climat. Il faut dire que son activité professionnelle est intimement liée aux saisons. Le transport des marchandises étant assujetti aux conditions de la navigation et à l'état des routes, le négociant doit donc bien observer les fluctuations météorologiques - vents, gel, enneigement, dégel - qui peuvent retarder ou accélérer l'arrivée de ses commandes. Par exemple, la formation du pont de glace reliant Québec à la rive sud rythme largement la vie économique. Juncken note: «quand la rivière prend, c'est-à-dire gèle de Québec à la pointe Lévis [...] ce n'est pas seulement l'occasion de divertissements mais de grands profits pour la ville, ainsi que pour les habitants de la rive sud, qui peuvent alors apporter leurs produits au marché en grandes quantités sans inconvénient. $\gg^{20}$ Mais derrière ces raisons terre-à-terre s'en trouve une autre, inconsciente mais néanmoins fondamentale, partagée par de nombreux auteurs de journaux. La notation quotidienne de la température répond à un besoin d'insertion dans l'environnement naturel. Comme il lui est impossible de contrôler les phénomènes météorologiques, le diariste cherche à les comprendre et à se les approprier. Comme le précise M. Motz:

20 Entrée du 4 décembre 1788. Traduction de l'auteure, comme dans toutes les citations qui suivent. 
Par ce classement des événements, cette recherche de la symétrie, l'écrivant tente de se placer lui-même ou elle-même en harmonie avec le monde naturel. En notant le temps qu'il fait au début de chaque entrée, le diariste se situe dans l'environnement naturel. ${ }^{21}$

Le journal évoque à plusieurs reprises cette communion avec la nature. De Charlesbourg, au nord de Québec, il observe le paysage: «sur le sommet des hauteurs, donnant la plus belle vue sur l'Ille d'Orléans, le fleuve [...] , Pointe Lévis, la ville de Québec, tout SainteFoy, Ancienne et Indienne Lorette, Cap Rouge, Beauport, la rivière Saint-Charles etc., tant que je crois qu'on ne peut trouver un plus bel endroit au Canada...» ${ }^{22}$

L'immigrant qu'est Henry Juncken doit aussi se construire rapidement une image mentale du plan de la ville. Les nombreuses promenades qu'il effectue lui permettent d'établir des repères physiques et de cartographier l'espace urbain. Une analyse minutieuse de l'ensemble de ses déplacements quotidiens montre que les sorties se partagent entre deux types de parcours qui se distinguent par l'heure et le lieu: un parcours de travail et un autre de loisir. Le parcours d'affaires de Juncken le mène invariablement à la Basse-Ville. Quittant sa maison située à l'angle des rues Saint-Jean et du Palais à la Haute-Ville, le marchand commence généralement sa tournée vers huit heures par une visite au marché de la place Notre-Dame, devant la cathédrale. Puis, il lui arrive souvent d'emprunter la côte de la Montagne pour se rendre au marché de la Basse-Ville près des quais et, de là, chez d'autres commerçants. Le retour s'effectue vers onze heures, en suivant le chemin inverse. Après le dîner, qui se prend vers treize heures, Juncken va encore parfois visiter des confrères. Le parcours d'affaires se termine avec son retour à la maison vers quatorze ou quinze heures.

C'est alors que débute normalement la promenade de loisir qui mène le marchand hors des sentiers battus. S'il sort avant le repas du soir, qui est servi vers dix-huit heures, il se rend habituellement chez le tavernier Shoemaker pour y jouer au backgammon avec son hôte. Les soirées du diariste sont consacrées aux visites chez ses amis et à des rencontres au «Merchant Coffee House» de la Basse-Ville. Les

21 Marilyn Ferris-Motz, «Folk Expression of Time and Place: 19th Century Midwestern Rural Diaries», Journal of American Folklore, 100,396 (avril-juin 1987): 145. Traduction de l'auteure.

22 «on the summit of the hill, commanding a most beautiful prospect of the Isle of Orleans, the River [...] , Point Levy, the City of Quebec, all St.Foy, ancient \& Indian Lorete, Cape Rouge, Beauport, the River St.Charles etc. so that I think a better place cannot be fined upon in all Canada...». Entrée du 19 février 1789. 
dimanches sont réservés à de longues promenades en ville qui lui permettent de rencontrer des gens d'autres milieux.

Quelle que soit la nature du parcours effectué, celui-ci est jonché de points de repère qui aident le promeneur à orienter ses déplacements: le palais épiscopal, situé au haut de la côte de la Montagne, alors loué et occupé par l'administration coloniale; les barraques du parc de l'Artillerie et la redoute Dauphine, où Juncken peut obtenir des lettres de change sur Londres sous la forme de demi-soldes d'officiers; les quais de Louis Dunière et de James Johnston, alors en construction, de même que Wolfe's Cove et le Cul-de-Sac où sont ancrés les navires; enfin, le «Merchant Coffee House» où marchands, capitaines de navires et artisans se rassemblent pour discuter des affaires du jour. D'autres jalons correspondent aux lieux où habitent ses amis, de Sainte-Foy à Québec en passant par Charlesbourg (Limoilou). Tous ces repères sont essentiels à la bonne marche de ses affaires; ils lui fournissent un répertoire de l'espace en le familiarisant avec la topographie de Québec.

Nous connaissons peu de chose sur la maison de Juncken. Seules sont mentionnées les pièces les moins privées: la boutique, la cuisine et la salle à manger au rez-de-chaussée; le salon à l'étage. Pourquoi cette discrétion sur son espace domestique et sur sa vie privée? $\mathrm{Ce}$ quasi-silence n'est peut-être pas étonnant dans le cas de Juncken. Faut-il rappeler qu'il mène une vie privée vraisemblablement équilibrée et sans problème. Ce n'est pas la vie familiale qu'il doit rétablir, mais bien une image publique ravagée par les malheurs vécus aux États-Unis. Et cette image publique se reconstitue par des contacts avec les gens que l'on invite à venir prendre le thé au salon, c'est-àdire en établissant avec eux de bonnes relations. Les quelques mentions que le diariste accorde à son espace domestique portent donc surtout sur les pièces socialement stratégiques. De même, nous notons une certaine préoccupation pour les foyers et le poêle, soit les lieux de rassemblement et d'intimité par excellence ${ }^{23}$. Ces détails en apparence anodins sont particulièrement révélateurs de l'attitude d'un homme qui se préoccupe constamment de son image et de son intégration sociale.

\section{L'IMAGE DE SOI: LE CORPS ET L'ESPRIT}

La majorité des auteurs de journaux personnels se préoccupent de leur santé24. Henry Juncken ne fait pas exception et on sent que son

23 Voir l'étude de Marcel Moussette, Le chauffage domestique au Canada: des origines à l'industrialisation (Québec, Presses de l'Université Laval, 1983).

24 Roy Porter, Disease Medicine and Society in England 1550-1860 (London, Macmillan, 1987), 28. 
journal personnel, par les nombreuses références à la santé, lui sert à dialoguer avec son corps. Il pratique beaucoup la marche, par hygiène et, comme beaucoup de ses contemporains, il est convaincu que la transpiration est le signe d'un sain exercice ${ }^{25}$. Les passages consacrés à ce sujet révèlent que l'intérêt du diariste pour la médecine est loin d'être superficiel: il prend la peine de noter le nom des médicaments prescrits ainsi que leur efficacité. Le marchand ne fait d'ailleurs confiance qu'à la médecine «institutionnalisée», celle des médecins reçus par le Bureau des examinateurs ${ }^{26}$. Il est sans conteste attiré par les membres de la profession médicale: il en nomme treize au long de son journal personnel, dont deux qui deviennent ses amis, soit J. G. Weiss (Le Blanc), chirurgien démobilisé du régiment allemand de Lorsberg, et un nommé Dillor ${ }^{27}$. Henry Juncken est bien conscient que la médecine ne guérit pas tous les maux. Contre la goutte qui l'afflige périodiquement, il ne consulte aucun médecin et ne recourt à aucun traitement ${ }^{28}$ car il sait que la science demeure impuissante face à cette maladie.

Après le corps et davantage encore, c'est le vêtement qui est utilisé comme un instrument de sociabilité ${ }^{29}$. L'analyse de sa garde-robe montre en effet comment Juncken cherche à se démarquer du peuple et à s'identifier au groupe des marchands britanniques. La seule concession vestimentaire au pays, ce sont les mocassins de fabrication amérindienne, qui lui sont nécessaires pour circuler dans les rues glacées. Les autres éléments du costume sont typiquement britanniques: les «breeches» (culottes) qui descendent jusqu'au genou, la chemise de flanelle, le gilet bien ajusté avec ses rayures verticales très populaire en Angleterre dans les années $1780^{30}$, le manteau long (great coat) avec ses grandes poches et le chapeau qui vient coiffer le tout.

Henry Juncken partage manifestement la culture de l'élite de la ville de Québec. Sa profession lui facilite l'accès à l'imprimé en lui fournissant de nombreux contacts avec des propriétaires et des dépositaires de livres et de journaux, les moyens financiers pour les acheter

25 À l'époque, les gens sont convaincus que pour se tenir en santé, il faut, entre autres, faire de l'exercice. Ibid., 25.

26 Jacques Bernier, La médecine au Québec. Naissance et évolution d'une profession (Québec, Presses de l'Université Laval, 1989), 1-2.

27 Diller, Tiller, Dillor et Tillor: ces variantes phonétiquement proches prêtent à confusion. Ce médecin est probablement Allemand lui aussi.

28 Entrées du ler au 15 septembre 1788 et du 25 avril 1789.

29 Christine Godin, "L'œuvre pionnière de Madeleine Doyon-Ferland», Canadian Folklore canadien, 10,1-2 (1988): 14.

30 C. Willet et Phillis Cunnington, Handbook of English Costume in the Eighteenth Century (Boston, Plays Inc., 1972). 
et, enfin, du temps libre pour les lire. À Québec, les marchands font en effet un usage important du livre. Dans son analyse des inventaires après décès, Yvan Morin démontre que chez les protestants, ce sont les marchands qui possèdent le plus de livres (29\%), suivis par les membres des professions libérales $(23 \%)^{31}$. La circulation des livres s'effectue grâce, entre autres, au système du prêt privé qui est alors très répandu, faute de bibliothèque publique. Échangé entre les membres de l'élite, l'imprimé devient un objet de socialisation qu'exploite régulièrement Juncken. Lui-même possède une traduction des Entretiens sur la pluralité des mondes, de Bernard Le Bovier de Fontenelle (1657-1757) $)^{32}$, et un atlas ${ }^{33}$ qui lui permettent de faire partie de ce réseau. Ainsi, le brasseur William George lui prête un livre d'astronomie en échange de ses Entretiens... ${ }^{34}$; Mathew Bell, alors jeune employé ambitieux du marchand Thomas Lee, lui laisse la traduction des six tomes des Mémoires de Sully que Juncken lit avidement $^{35}$. Il est aussi un fidèle lecteur de l'Almanach de Québec et des journaux locaux: La Gazette , $^{36}$ et le Quebec Herald, Miscellany and Advertiser ${ }^{37}$.

Le journal dévoile un homme qui se veut profondément religieux et compatissant envers la misère humaine. Il vient en aide à ses concitoyens dans le besoin en participant à l'"Amicable Society», une société essentiellement philanthropique qui recueille des fonds pour subvenir aux besoins des familles démunies. Juncken fut son premier président. Son dévouement est remarquable dans l'affaire du capitaine Gale qui, reconnu coupable de meurtre, attend son exécution les fers aux pieds ${ }^{38}$. Juncken lui procure de la nourriture et note les conditions déplorables de sa détention: «pauvre homme, il nous a envoyé un mot nous priant de lui procurer quelque chose car il meurt de faim.» ${ }^{39} \mathrm{Il}$ accepte de fournir jusqu'à concurrence de quatre guinées pour l'entretien du condamné $e^{40}$, et, une fois la bourse épuisée, il s'occupe lui-même de $\mathrm{Gale}^{41}$, va le voir en prison et lui apporte des pommes ${ }^{42}$.

31 Yvan Morin, Les niveaux de culture à Québec 1800-1819. Étude des bibliothèques privées dans les inventaires après décès, thèse de M.A., Université Laval, 1979, 80.

32 Entrée du 14 novembre 1788.

33 Entrée du 18 mars 1789.

34 Entrée du 14 novembre 1788.

35 Entrée du 18 mars 1789.

36 Acheté le 23 décembre 1788.

37 Achat des deux premiers numéros le ler décembre 1788.

38 Entrée du 15 octobre 1788.

39 «poor Fellow he send us a few lines this morning begging that we might sent him something for he was in a Starving condition.» Entrée du 5 décembre 1788.

40 Entrée du 18 novembre 1788.

41 Entrée du 22 février 1789: «dont want to let him suffer in Confinment.»

42 Entrée du 21 novembre 1788. 
$\mathrm{Si}$, dans sa vie sociale, Juncken se montre débordant de générosité, il l'est beaucoup moins dans le domaine des affaires. Son attitude professionnelle peut se résumer par l'adage «les affaires sont les affaires». Entamer des poursuites contre un débiteur, refuser de faire crédit et exiger des garanties pour les prêts ne sont que quelques exemples des pratiques que le marchand doit couramment utiliser.

\section{LA VISION DE L'AUTRE: STRATÉGIES DE SOCIABILITÉ}

La vie sociale du marchand se divise en deux parties selon qu'il parle de sa famille ou de ses amis. Ces deux mondes, intérieur et extérieur, n'ont pas le même poids dans son journal: la vie avec les proches demeure à l'arrière-plan alors que la vie sociale extérieure occupe presque toute la scène du quotidien.

Sa famille, composée de sa femme Anna Barbara et de ses deux neveux, John et Peter Rees, n'est évoquée qu'en filigrane. Juncken ne parle pas des gens qui vivent à ses côtés, sans doute parce qu'il les connaît bien; il n'a pas à les analyser ni à les mettre en scène ${ }^{43}$. Les rares allusions du diariste à sa femme et à ses neveux ne permettent même pas de connaître leur âge.

La présence de l'épouse est évoquée uniquement lorsqu'il parle de ses ennuis de santé et de leurs promenades dominicales. Les neveux Rees n'attirent pas plus son attention: il note seulement les heures de leurs entrées et sorties ${ }^{44}$. C'est là-dessus qu'il termine généralement la notation quotidienne du journal, avant de se coucher. Juncken joue le rôle du chef de famille, responsable des individus qui habitent sous son toit et attentif à leur santé et à leurs allées et venues.

Les descriptions de la facette extérieure de sa vie sociale sont beaucoup plus nombreuses. Il décrit en moyenne cinq rencontres par jour ${ }^{45}$. Les individus qui forment son réseau de sociabilité professionnelle sont principalement des Anglais, mais on y trouve également des Écossais, des Irlandais, des Américains et quelques rares Canadiens français. Vers 1790, les anglophones forment près de $20 \%$ de la population de Québec ${ }^{46}$. Le monde des affaires et l'administration coloniale sont entre leurs mains, d'où la nécessité d'établir et d'entretenir de bons et de fréquents contacts avec eux. Quant aux rapports

43 Foisil, «L'écriture du for privé», Philippe Ariès et Georges Duby, dir., Histoire de la vie privée (Paris, Ed. du Seuil, 1986), 3: 346.

44 Des notes telles que «John \& Pcter sont allés chez Biker en soirée \& ils... rentrèrent vers 10 heures» sont courantes. Entrée du 4 octobre 1789.

45 Soit 1339 rencontres en 273 jours.

46 John Hare, La pensée socio-politique au Québec 1784-1812: analyse sémantique (Ottawa, Éditions de l'Université d'Ottawa, 1977), 11. 
avec les «french inhabitants», Henry Juncken ne fait des affaires qu'avec les plus prospères et les plus en vue tels que les frères Hamel de Sainte-Foy et le marchand et constructeur de navires Louis Dunière de Québec. La langue ne peut être considérée comme un obstacle insurmontable puisque, de son propre aveu, Juncken se débrouille en français ${ }^{47}$. Nous observons qu'il fréquente autant d'Allemands que de Canadiens français et recherche bien davantage la compagnie de ses compatriotes. David T. Ruddel a bien montré d'ailleurs qu'après la Conquête l'ethnicité devint un critère de sélection pour l'emploi et pour les relations sociales ${ }^{48}$.

Les contacts d'ordre professionnel ou rendez-vous d'affaires ne représentent que le quart des rencontres notées dans le journal de Juncken. Il pratique surtout une sociabilité du loisir marquée par des relations qui oscillent entre deux extrêmes, allant de la simple courtoisie - la "common civility» ${ }^{49}$ - à l'amitié très étroite. Son intérêt pour la santé et la médecine l'aide aussi à conforter sa position sociale. Henry Juncken est toujours ravi de rencontrer l'examinateur du bureau des médecins, le docteur Frederick William Oliva ${ }^{50}$, «very portey \& polite», qui possède un large réseau de connaissances et ouvre au marchand la porte du milieu des professions libérales. L'analyse de ses amitiés nous révèle les bases ou les valeurs sur lesquelles elles s'appuient. Les paroles échangées et les activités partagées forment un miroir dans lequel se reflètent les attentes de Henry Juncken face à l'Autre et aussi les limites de ces amitiés. Celles-ci tournent autour de six hommes et comprennent trois types de relations, chacune révélant une facette de la stratégie d'intégration de l'étranger.

Henry Juncken est féru de connaissances et affiche un grand intérêt pour les sciences et la culture en général. Il se donne beaucoup de mal pour susciter et entretenir une amitié solide avec le maître d'école John Jones et le médecin allemand J. G. Weiss. Il partage avec le premier son intérêt pour l'arithmétique et l'astronomie, de même que pour les délicates questions de théologie. Pour consolider cette amitié toute récente, Juncken seconde Jones dans la préparation de l'examen public de ses élèves ${ }^{51}$ et, plus tard, il lui fait cadeau d'une volaille ${ }^{52}$. Le second, Weiss, échange son savoir médical avec le marchand qui

47 Entrée du 9 février 1789.

48 Courrier de Québec, 11 avril 1807, John Hare, op. cit., 41.

49 Entrée du 31 mai 1789.

50 Pour plus de détails sur Oliva, voir F. Murray Green, «Frederick-William Oliva», Dictionnaire biographique du Canada, IV: 638-639.

51 Entrée du 24 décembre 1788.

52 Entrée du 27 janvier 1789. 
lui demande de soigner un de ses amis, le teinturier Samuel Casey, et, par la suite, les membres de sa propre famille: sa femme, pas moins de quatre fois ${ }^{53}$, son neveu Peter Rees également quatre fois ${ }^{54}$ et son domestique Vallentin à deux reprises ${ }^{55}$. Entièrement satisfait des services de Weiss, Juncken finit par se rendre lui-même chez lui pour se faire extraire une dent ${ }^{56}$. Pour le marchand, ces doctes amitiés lui offrent l'occasion de se lier avec des membres respectés de la société britannique.

En bon citoyen, le marchand se porte à la défense de principes humanitaires et il se montre sensible à la souffrance d'autrui. Il ne ménage pas ses efforts et ses démarches pour secourir un Samuel Casey pauvre et alcoolique. Tout au long de l'automne et jusqu'au printemps, Juncken soutient moralement et financièrement la famille de Casey et sollicite même des contributions parmi ses confrères et amis. Peu après le décès de la femme de Casey, il aide ce dernier à vendre ses biens pour payer ses dettes afin de pouvoir retourner en Angleterre. Le diariste joue également le rôle de soutien moral auprès de son voisin Biker que le rhumatisme tient presque continuellement cloué au lit. Comme le vieillard vit seul avec son épouse, Juncken se fait un devoir de le visiter régulièrement, de lui offrir de petits présents ${ }^{57}$ et de l'inviter à dîner. Ce type de relation lui permet de se montrer généreux et de construire une image publique séduisante qui peut lui attirer une certaine reconnaissance de la part du groupe qu'il privilégie.

Henry Juncken fait preuve de fidélité envers ses anciens amis. Les liens qui l'unissent au cordonnier Samuel Jeffrey trouvent leurs racines dans un passé commun. En effet, les deux hommes ont habité Philadelphie; ils ont tous deux connu les cellules de la prison de Reading et ils arrivent à Québec à quelques mois d'intervalle ${ }^{58}$. C'est vraisemblablement sur les conseils de Jeffrey que Juncken s'est installé à Québec. Samuel Jeffrey compte-t-il sur ces liens pour emprunter souvent de l'argent à Juncken et pour le rembourser avec d'importants retards, pouvant aller jusqu'à six mois? Jeffrey, misant

53 Pour une purge le 12 octobre 1788 et d'autres traitements non précisés les 18 octobre 1788, 23 octobre 1788 et 27 mai 1789.

54 Deux saignées, la première le 23 octobre 1788 et la seconde le $1^{\text {er }}$ février 1788 , une purge le 18 octobre 1788 et un vomitif le 25 octobre 1788.

55 Entrées du 20 et 21 février 1789.

56 Entrée du 18 février 1789.

57 Entrée du 16 décembre 1788.

58 La présence de Samuel Jeffrey à Londres est attestée par sa demande de dédommagement pour pertes subies lors de la guerre. ANC, MG 14, Audit A-6, 13: E-71; 2: 115. 
sur la solidité de leur amitié et sachant à quel point le marchand soigne son image publique, devine que ce dernier n'osera pas se montrer trop empressé de recouvrer son dû. Mais la prudence a des limites et, malgré l'amitié qui le lie au cordonnier, Henry Juncken finit par réclamer son argent. Il ira même jusqu'à saisir un arrivage de cuir chez Jeffrey. Il reste que peu de débiteurs sont parvenus à faire patienter Juncken aussi longtemps que Samuel Jeffrey.

\section{DE L'AUTRE CÔTÉ DU MUR: LES «FRANÇAIS»}

Lorsque Henry Juncken s'installe à Québec, il découvre une communauté britannique vivant au sein d'une majorité canadiennefrançaise ${ }^{59}$. Le diariste observe de près les coutumes de ces «french inhabitants». Sa perception change selon qu'il se réfêre à un individu en particulier ou au groupe ethnique dans son ensemble. Il prend la peine de se lier avec ceux bien en vue comme la famille Hamel de Sainte-Foy, la plus riche de cette paroisse selon lui ${ }^{60}$. D'autres relations doivent leur existence à la curiosité intellectuelle du marchand, comme celle avec le maître d'école Neville que Juncken admire pour ses qualités d'homme de lettres ${ }^{61}$.

Le diariste n'hésite pas à nouer des rapports avec des curés cultivés pour satisfaire ses goûts intellectuels. En février, il va dîner chez le curé Borel de Sainte-Foy, "qui est naturellement cultivé» ${ }^{62}$. Le marchand fait certainement bonne impression sur son hôte car ce dernier lui rend aussi visite quelques jours plus $\operatorname{tard}^{63}$. Mais la palme revient au curé de Charlesbourg qui a vraiment su charmer le marchand qui écrit:

J'allai... chez monsieur le curé Tremain, qui m'était complètement étranger. Il m'entretint avec une véritable culture, m'invita à dîner, ce que j'acceptai. Il se montra un parfait gentilhomme, à tel point que je suis extrêmement heureux de le connaître, et l'ai invité à venir chez moi, ce qu'il a accepté. ${ }^{64}$

59 John Hare, op. cit., 11. La distinction étant entre les anciens sujets par opposition aux nouveaux sujets, qui le sont devenus au moment de la Conquête.

60 Entrée du 16 novembre 1788. Il prend la peine de préciser: «Michel Amel and his wife from Ste-Fois were at our house they are the wealthyest family of that Parishe and this is the first time that they were at our house.»

61 Entrée du 26 février 1789. "Could not but admire for he writes a most excellent hand, and now by the conversation found that he is an excellent scholar \& a perfect grammarian, so that he is exceeding well qualifyed for a schoolmaster.»

62 Entrée du 10 février 1789. "...who is naturally polite.»

63 Entrée du 19 février 1789.

64 Entrée du 21 février 1789. 
Individuellement donc, les Canadiens français peuvent posséder des qualités ${ }^{65}$. Ses commentaires sur certains d'entre eux, tel le prospère cultivateur Louis Hamel, sont carrément flatteurs: «plein de compliments, à la manière française, il m'a semblé aussi intelligent qu'un autre.» ${ }^{66}$ En doutait-il? Ailleurs il note: «une volubilité de langage, propre aux Françaises. ${ }^{67}$ Enfin, Juncken admire le courage et l'endurance des Canadiens français qui osent traverser le fleuve en hiver:

J'arrêtai un moment près de la grande Batterie et regardai le fleuve, et le pays alentour qui semblait vraiment très beau. Le fleuve est couvert de glaces coupées par de larges étendues d'eau. Les canots passent entre les glaces, puis sont tirés sur la glace et remis à l'eau. Pour le spectateur, cela semble vraiment dangereux mais les Français n'y voient aucun danger et prennent un grand plaisir à épuiser ainsi leurs forces. ${ }^{68}$

Cependant, les Canadiens français comme groupe ethnique lui déplaisent et il ne s'en cache pas. Il les considère peu serviables. Lorsque l'un d'eux l'aide à transporter un mouton qu'il vient d'acheter au marché, il note que l'homme refuse tout dédommagement: «sans accepter une pièce ou rien d'autre pour sa peine, ce qui est rare chez un Français.» ${ }^{69} \grave{A}$ un autre moment, il dénonce l'insoumission des Canadiens français qui gêne le bon déroulement du commerce:

J'allai au marché d'en haut. Il y avait beaucoup de provisions compte tenu la saison, mais chères. Les habitants français sont vraiment réfractaires à l'idée de ranger leurs voitures selon le nouveau règlement pour prévenir les cahots, et c'est la raison pour laquelle si peu viennent au marché, et ceux qui viennent vendent leurs provisions à un prix élevé. ${ }^{70}$

Mais le plus grand défaut des Canadiens français est qu'ils sont catholiques romains ${ }^{71}$. Les commentaires du marchand sur les catholiques en tant que groupe sont mordants, voire méprisants. Lors des

65 Entrée du 9 février 1789. Il emploie les qualificatifs suivants: compétent, obligeant et bien élevé.

66 Entrée du 21 février 1789. «Full of compliments after the french manner, he seemed me to be as clever as a man of this name.»

67 Entrée du 26 mars 1789. "A volubility of language, for beeing a french women.»

68 Entrée du 7 janvier 1789.

69 Entrée du 3 décembre 1788. «the Man was very kind... called and left the sheep without accepting a Dram or any thing clse for his trouble, which is rare in a frenchman.»

70 Entrée du 6 décembre 1788.

71 Nous ignorons quelle est la religion de Juncken. Il n'est pas presbytérien comme ses neveux Rees. Il n'assiste que quatre fois à l'office anglican en neuf mois et son inhumation par cette Église en 1802 ne prouve pas son appartenance à cette religion. Compte tenu de ses origines, il est possible qu'il soit luthérien. 
festivités entourant le Mardi gras, il observe les moeurs des catholiques qui, juste avant le début du Carême, se déguisent et font bombance: «ils sont libres de se gaver [...] autant qu'ils en peuvent avaler... car demain le Carême commence et ils devront jeûner pendant quarante jours. $\gg^{72}$ On le voit, ce n'est pas l'ignorance des pratiques et des rites catholiques qui sont à la base de ce mépris. Le ton est parfois moqueur. Ainsi lorsqu'il décrit: «une grande procession de catholiques romains allant à la cathédrale demander à Dieu de bénir les fruits de la terre», il ajoute: «Si cela donne quelque chose, nous en profiterons aussi.» ${ }^{73}$ Pour Juncken, les catholiques ne sont qu'un groupe de «papistes» qui se réunissent à l'église avec «les autres saints ${ }^{74}$ pour faire leurs dévotions. S'il apprécie l'intelligence de certains individus, il considère toutefois le groupe comme ignorant, le rôle du curé étant celui de "grand Oracle d'un peuple ignare» ${ }^{75}$. L'opinion du marchand rejoint celle de ses compatriotes anglophones, dont la stratégie rhétorique, comme le souligne Maurice Lemire, consiste «à insister sur l'ignorance des Canadiens pour montrer qu'ils peuvent devenir le jouet d'une faction malintentionnée qui veut ni plus ni moins leur perte» ${ }^{76}$.

\section{CONCLUSION}

Plutôt que de peindre un tableau quelque peu statique de la vie quotidienne de l'époque ou de la mentalité de l'écrivant, il nous semblait beaucoup plus intéressant d'essayer de comprendre comment la pratique des représentations par l'écriture d'un journal contribue au fonctionnement social de l'individu. Dans le cas de Henry Juncken, nous avons surtout porté attention aux représentations de l'espace physique, de soi et de l'Autre. L'analyse a dévoilé un homme soucieux de son image publique, image qu'il soigne par des comportements bien mesurés et par des attitudes philanthropiques, le tout dans le but d'éviter de s'attirer l'animosité des membres de sa communauté d'accueil et de cesser d'être perçu comme un étranger.

72 Entrée du 24 février 1789. «they are at Liberty to stuff themselves, with at as much fat $\&$ most as they can cram down their throat, for to morrow Lent begins, when they must fast for forty Days.»

73 Entrée du 19 mai 1789. «a grand procession of the Roman Catholick going into the cathedral ... to ask a blessing to ... to prosper the fruits of the earth. If it avails any thing we shall have a share of it.»

74 Ibid., «the rest of the saints».

75 Entrée du 21 mars 1789. «the grand Oracle of a set of ignorant People.»

76 Maurice Lemire, "Savoir et pouvoir: le cas du Bas-Canada», Questions de culture. Vol. 1: Cette culture que l'on appelle savante (Québec, Institut québécois de la recherche sur la culture/Leméac, 1979): 68. 
Le grand soin que prend le diariste à noter tous ses déplacements, mettant à nu sa vie du dehors, dévoile toute l'attention qu'il porte à son image publique. L'espace privé est en marge du journal personnel. Il sert de cadre aux activités du marchand, professionnelles ou non professionnelles, mais demeure en filigrane. Juncken ne semble pas accorder une grande importance à son intérieur domestique. En fait, dans la logique de son journal personnel, de tels détails sont superflus, tout comme ceux sur sa famille. Son journal manifeste la recherche d'une identité publique conforme à celle exigée par sa société adoptive.

Il n'y a pas de doute que Henry Juncken vise l'intégration dans l'élite britannique de Québec. Pour lui, étranger chez les étrangers, il n'est pas question de s'intégrer aux «french inhabitants», ces gens «ignorants» et «bigots». Ici les contacts se résument à ceux qui offrent quelque chose en retour, que ce soit une conversation agréable ou une provision de bois de chauffage. Ce n'est pas en côtoyant les Canadiens français que Juncken pourra s'intégrer à la bonne société britannique et échapper à une certaine marginalité sociale que lui confère son statut de réfugié.

Le journal personnel est, en fait, une manifestation des efforts d'intégration du diariste. Le regard qu'il jette sur le monde environnant est conditionné par son désir de protéger sa vie publique. Il cherche à bien connaître les us et coutumes du pays pour ne pas enfreindre des lois informelles, mais bien présentes, et risquer ainsi l'exclusion. Mais surtout, le journal personnel que nous a laissé Johann Henrich Juncken est le fait d'un homme qui, n'ayant pas de racines dans son nouveau milieu, a recours à un journal pour se mettre en scène, analyser ses paroles et ses gestes, étudier son comportement et appliquer une stratégie d'intégration. Donc, son journal est plus qu'un simple manuscrit: c'est une partie de la conscience de son auteur et un instrument précieux de sa sociabilité. 